\title{
Impact of Modification Rate in Artificial Bee Colony for Engineering Design Problems
}

\author{
Tarun Kumar Sharma \\ Amity Institute of Information Technology, Amity University Rajasthan, India \\ E-mail: taruniitr1@gmail.com \\ Millie Pant \\ Department of Applied Science Engineering, Indian Institute of Technology Roorkee, India \\ E-mail: millidma@gmail.com \\ Deepshikha Bhargava \\ Amity Institute of Information Technology, Amity University Rajasthan, India \\ E-mail: dbhargava1@jpr.amity.edu
}

\begin{abstract}
Artificial Bee Colony (ABC), a recently proposed population based search heuristics which takes its inspiration from the intelligent foraging behavior of honey bees. In this study we have studied the impact of modification rate (MR) in basic $\mathrm{ABC}$ by gradually increasing it from 0.1 to 0.9 . This impact is studied on four engineering design problems taken from literature. The simulated results show that it is beneficial to set the modification rate to a lower value.
\end{abstract}

Index Terms - Artificial Bee Colony, Modification Rate, Engineering Design Problems, Optimization.

\section{Introduction}

ABC, proposed by Karaboga in 2005 [1] is a relatively recent bio-inspired metaheuristic optimization algorithm that mimics the natural intelligent foraging behavior of honey bees. Initially $\mathrm{ABC}$ was proposed and developed for solving continuous optimization problems [2] [3]. Since the introduction of $\mathrm{ABC}$, it has also been applied to solve a wide variety of optimization problems arising in various disciplines [4][12]. The detailed survey can be studied in [13]. ABC comprises of three kinds of bees namely (a) Scout (b) Employed and (c) Onlooker bees. The bees intelligently organize themselves and divide the labor to perform the tasks like searching for nectar, sharing information about the food source etc.

The position of a food source represents a possible solution to the optimization problem and the nectar amount of a food source corresponds to the quality (fitness) of the associated solution. A brief overview and mathematical model of $\mathrm{ABC}$ is presented in section 2.

In this paper we have studied \& analysed the impact of modification rate in basic ABC while solving four engineering design problems. This paper is structured as follows: Parameter settings are briefed in Section 3 and analyses of the impact of modification rate in $\mathrm{ABC}$ are discussed in Section 4. Finally the conclusions are presented in Section 5.

\section{Outline of $\mathrm{ABC}$}

\section{Foraging behavior of Bees:}

In the initial phase of foraging, bee explores the environment randomly in search of food sources. When the forager bee finds the food source it becomes employed bee. After exploiting the discovered food source, employed bee returns to the hive and unloads the gathered nector. Now it's up to bee to go back directly to the discovered food source or can share the site information to the onlooker bees waiting in the hive by performing a dance called waggle dance on the dance area. Through this dance the employed bee informs the onlooker bee about the direction w.r.t. Sun, distance and quality (Fitness) of the food source. If the food source exploited by employed bee gets exhausted then the bee becomes scout bee, also called the replacement mechanism, and explores the new food sources randomly. The Onlooker bees choose the source site depending on the frequency of the dance, as frequency is proportional to quality of the food source. This is how the onlooker bee becomes the employed bee.

In $\mathrm{ABC}$ algorithm the food Sources represents the candidate solutions/possible solutions and the nector amount of the food source represents the fitness associated with the solution for the optimization problem. And each of the food sources is exploited by only one employed bee hence the numbers of food sources are equal to the employed bees.

By this analogy between the intelligent foraging behavior of bees and the ABC algorithm, the basic ABC algorithm can be explained. This is also described using a graphical representation in Fig. 1. 


\subsection{Unconstrained $A B C$}

$\mathrm{ABC}$ tries to model natural behavior of real honey bees in food foraging. The colony of artificial bees comprises of three groups of bees: employed bees which takes the responsibility for exploiting the food sources and communicate the information to the onlooker bees, which are waiting in the hive. Onlooker bees chooses the food sources by watching the waggle dance performed by employed bees while scouts explores the food sources randomly based on some external clue.

The basic steps of unconstrained $\mathrm{ABC}$ are discussed below:

1. Initialization of Food Sources (Population of Solutions): The first step of $\mathrm{ABC}$ is generation of initial population. Suppose, the initial population consists of $S N$ number of $n$-dimensional real-valued vectors, representing the food sources, which are generated randomly. Suppose $X_{i}=\left\{x_{i, 1}, x_{i, 2}, \ldots, x_{i, n}\right\}$ denotes the $i^{\text {th }}$ food source in the population, where each food source is initialized as:

$$
x_{i, j}=x_{\min , j}+\operatorname{rand}(0,1)\left(x_{\operatorname{mxx}, j}-x_{\min , j}\right)
$$

Where $i \in 1,2, . ., S N ; j \in 1,2, \ldots, n . x_{\max , j}$ and $x_{\min , j}$ denotes the upper and lower bound constraints respectively of decision variable. These food sources are assigned randomly to $S N$ number of employed bees and their fitness is determined.

2. Determination of new food locations (Employed Bees Stage): The duty of employed bees is to determine new food source say, vi with the help of the food source xi assigned to it during the initialization phase. The employed bees exploit the food source. The equation used is:

$$
v_{i j}=x_{i j}+\phi_{i j}\left(x_{i j}-x_{k j}\right)
$$

Where $k \in\{1,2, \ldots, S N\}$ and $j \in\{1,2, \ldots, n\}$; Control parameter $\phi_{i j} \in[-1,1]$ gives the step length which decides the movement of bees. $k$ is generated randomly such that, $k \neq i$. After a new food source has been generated by the employed bee, a tournament selection is held between $v_{i}$ and $x_{i}$ and the one with a better fitness value becomes the member of the population.

3. Evaluation of Nector Information or quality of solution (Onlookers Phase): The work of onlookers is to enhance the exploration capability of $\mathrm{ABC}$. An onlooker evaluates the nectar information (i.e. the fitness of solutions) collected from all the employed bees and selects a food source xi using probability Pi as:

$$
P_{i}=f_{i} / \sum_{k=1}^{S N} f_{k}
$$

Where $f_{i}$ denotes the fitness value of the $i^{\text {th }}$ food source. The onlooker after selecting the food source $x_{i}$, modifies it by using equation (2). Once again a tournament is held between the food source selected and the food source modified by the onlooker and once again as in previous step, the one with better fitness value becomes a member of the population. Therefore, good food sources attract more onlooker bees compared to bad ones.

Diversity mechanism in ABC (Significance of Scouts): The main work of scouts is to induce diversity in $A B C$. An employed bee which is not able to improve the fitness value of a food source becomes a scout. A scout is activated when it is observed that the fitness of a food source $x_{i}$ shows no betterment even after a specified number of trials limit. Stagnation in the fitness value of a food source indicates that the particular food source may be replaced with a new food source. Scout produces a new food source with the help of equation (1).

Pictorial representation is shown in Fig. 2.

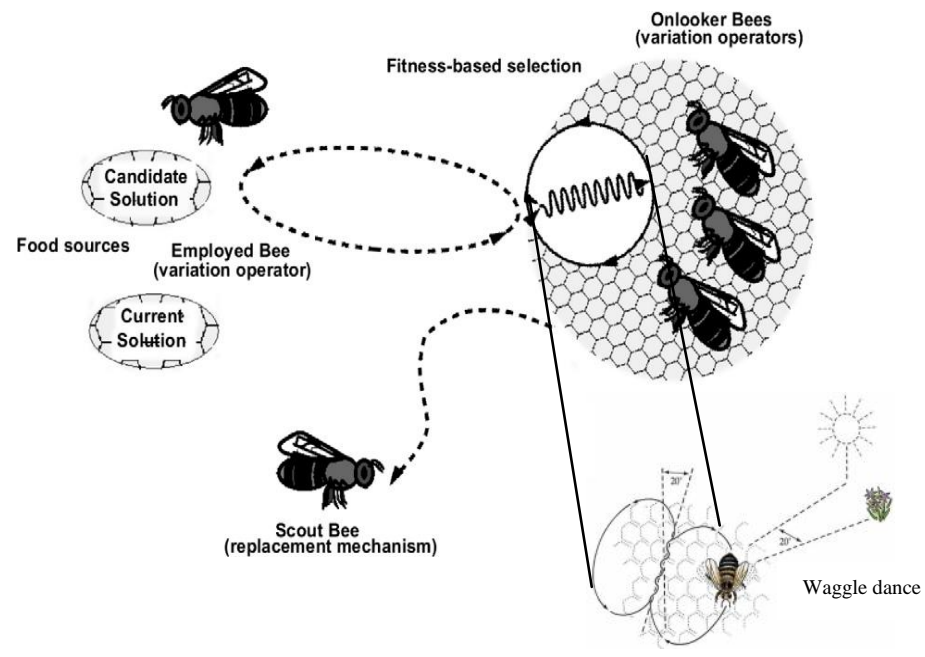

Figure 1. Graphical representation of the elements of $\mathrm{ABC}$ algorithm 


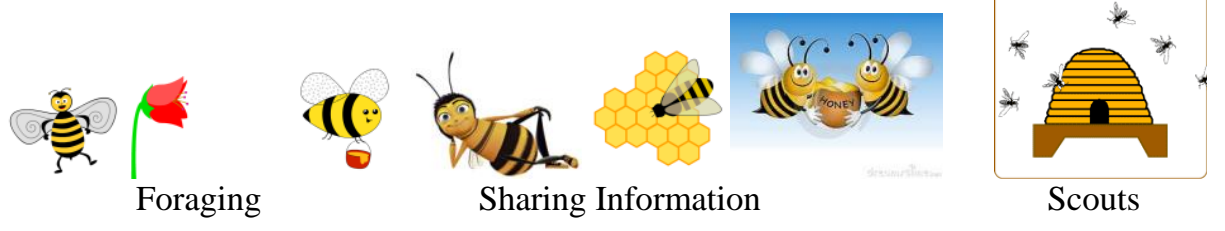

Figure 2. Bee Colony food foraging and sharing process

\subsection{Constrained $A B C$}

ABC, with small changes, can easily be modified for dealing with problems having constraints as well [[13]]. In this method a user defined control parameter called modification rate (MR), is introduced. With the help of $\mathrm{MR}$, it is decided stochastically whether a food source xi should be retained or not. It given by the equation:

$$
\begin{aligned}
& \text { If Rj } \leq M R \\
& v_{i j}=x_{i j}+\phi_{i j}\left(x_{i j}-x_{k j}\right) \\
& \text { Else } \\
& v_{i j}=x_{i j}
\end{aligned}
$$

Where, Index $\mathrm{k} \in\{1,2, \ldots, S N\}$ - randomly chosen such that $k \neq i ; j \in\{1,2, \ldots, n\} . R_{j}$ is generated randomly between 0 and 1in each iteration; while $M R$ has a fixed value predefined by the user in the beginning of the program.

To study the impact of $\mathrm{MR}$ in $\mathrm{ABC}$, the problem constraints are handled by following Deb's [[14]] three feasibility rules as criterion to compare solutions (food sources). They are described as follows:

- If there are two possible feasible food positions then one having higher nector (fitness value) is selected.

- If only one food position is feasible and other is infeasible then the one having feasible position is chosen.

- If both the food positions are infeasible then the one which violates less constrains is considered for the possible food position.

These rules bias feasible food sources over infeasible food sources. And there is no need of penalty parameter because a pairwise comparison (tournament selection) is done in this method. rand (), inbuilt function in $\mathrm{C}++$ is used to generate random food sources. The seed remains the same for every algorithm.

\section{Importance of Modification Rate}

An employed bee produces a modification (equation (4)) on the position of the food source (solution) in her memory depending on the local information (visual information) and evaluates the nectar amount (fitness value, quality) of the new source (new solution). As it can be seen from (equation (4)), the difference between perturbation on the position xij decreases. Thus, as the search approaches to the optimum solution in the search space, the step length is adaptively reduced. The first modification of $\mathrm{ABC}$ algorithm arises here. In the unconstrained version of $\mathrm{ABC}$ algorithm, only one randomly chosen parameter xij is modified while other parameters except for xij are copied from the old solution Xi. However, in the modified ABC algorithm, for each parameter xij, a uniformly distributed random real number, $(0 \leq R j \leq 1)$, is produced and if the real number is less than the control parameter value, modification rate (MR), and the parameter $\mathrm{xij}$ is modified. Here, we ensure at least one parameter to be modified if no parameter is changed.

\section{Parameters Settings for Simulation}

A test bed of four constrained engineering design optimization problems have been taken from the literature (also given in Appendix -I) to evaluate the impact of modification rate in basic ABC. The considered engineering optimization problems are F1: Welded beam design [[15]], F2: Pressure Vessel design [[16]], F3: Speed Reducer design [[17]], F4: Tension/compression spring design [[18]].

Artificial bee colony algorithm has been coded in $\mathrm{C}++$ and executed in Linux environment on dual core processor with 4GB RAM.

$\mathrm{ABC}$ has three control parameters: Food sources $(S N)$; limit for abandonment i.e. value of limit; and maximum cycle numbers $(M C N)$ and are defined as follows:

- The number of bees or colony size $(S N)$ that consist of equal number of employed as well as onlooker bees, is taken as 40 .

- Limit that controls the number of trials for the improvement of food source is taken as $S N^{*} n$, where is $n$ is number of decision variables. Food source is abandoned by its employed bee if it could not be further improved in defined number of trials of limit.

- Maximum cycle numbers are the number of cycles taken by bee for foraging the food sources and is fixed to 5000, which as also a termination/stopping criterion.

All the experiments were run 25 times i.e. algorithm is executed 25 times for each problem with a total of 5,000 objective function evaluations per run. 


\section{Analyses of the Impact of Modification Rate In ABC}

Modification rate (MR), is a new control parameter that has been added to the structure of basic Artificial Bee Colony algorithm by Karaboga and Basturk. The modification in parameter $x_{i j}$ (equation (4)), to be or not to be is controlled by the control parameter MR. In this study we tried to analyze the impact of this control parameter in optimization. For this analysis we have considered a small test bed of four engineering design problems taken from the literature. During the analysis the value of MR is gradually increased starting from 0.1 to 1.0 with an interval of 0.1 . The results obtained in terms of function fitness value, best, worst, mean and standard deviation of the test problems are presented in Table 1 - Table 4.

It has been analysed as the value for the modification rate increases, the probability of changing the optimization parameter $\mathrm{xj}, \mathrm{j}=1,2, \ldots, \mathrm{N}$ is increased. It can be considered that if modification rate take the smaller value we are looking for a new food source in the closer neighborhood. Otherwise, parameter takes the higher value we are looking for a new food source in the further neighborhood. The preliminary experiments show that it is beneficial to set the modification rate to a lower value between $0.4-0.6$. The function fitness value, mean value, worst and best value are plotted for all the four problems and shown in Fig. 3(a) to 3(d), where A represents the cost or fitness function curve, $B$ represents the curve consist of the mean values in each run, best and worst are the best and worst fitness values in all the runs and Min Mean is the minimum mean in all the runs.

TABLE 1. SIMULATION RESULTS OF WELDED BEAM DESIGN FOR MR = 0.1 TO 0.9

\begin{tabular}{|c|c|c|c|c|c|c|c|c|c|}
\hline MR & 0.1 & 0.2 & 0.3 & 0.4 & 0.5 & 0.6 & 0.7 & 0.8 & 0.9 \\
\hline$f(x)$ & 1.767963 & 1.745251 & 1.742464 & 1.738848 & 1.744395 & 1.736346 & 1.729843 & 1.728945 & 1.724902 \\
\hline Best & 1.767963 & 1.745251 & 1.742464 & 1.738848 & 1.744395 & 1.736346 & 1.729843 & 1.728945 & 1.724902 \\
\hline Mean & 1.963784 & 1.98587 & 1.883966 & 1.879168 & 1.926924 & 1.849995 & 1.877182 & 1.798791 & 1.78615 \\
\hline Worst & 2.39207 & 2.411513 & 2.289949 & 2.210244 & 2.486686 & 2.21497 & 2.330383 & 1.962422 & 2.13123 \\
\hline Std. Dev. & 0.149677 & 0.166706 & 0.153873 & 0.108806 & 0.174692 & 0.109368 & 0.137987 & 0.067238 & 0.082906 \\
\hline
\end{tabular}

TABLE 2. SIMULATION RESULTS OF PRESSURE VESSEL FOR MR = 0.1 TO 0.9

\begin{tabular}{|c|c|c|c|c|c|c|c|c|c|}
\hline MR & 0.1 & 0.2 & 0.3 & 0.4 & 0.5 & 0.6 & 0.7 & 0.8 & 0.9 \\
\hline$f(x)$ & 6152.253 & 6095.73 & 6108.776 & 6097.366 & 6094.628 & 6072.972 & 6066.236 & 6060.525 & 6060.373 \\
\hline Best & 6152.253 & 6095.73 & 6108.776 & 6097.366 & 6094.628 & 6072.972 & 6066.236 & 6060.525 & 6060.373 \\
\hline Mean & 6412.881 & 6273.631 & 6267.846 & 6260.437 & 6201.243 & 6171.936 & 6124.353 & 6189.578 & 6183.321 \\
\hline Worst & 6774.41 & 6490.634 & 6507.262 & 7208.776 & 6378.966 & 6390.021 & 6176.579 & 6569.961 & 6485.87 \\
\hline Std. Dev. & 219.1478 & 140.0591 & 125.6191 & 320.1834 & 105.3373 & 93.5339 & 37.38575 & 175.2342 & 135.8141 \\
\hline
\end{tabular}

TABLE 3. SIMULATION RESULTS OF TENSION COMPRESSION SPRING FOR MR = 0.1 TO 0.9

\begin{tabular}{llllllllll}
\hline MR & $\mathbf{0 . 1}$ & $\mathbf{0 . 2}$ & $\mathbf{0 . 3}$ & $\mathbf{0 . 4}$ & $\mathbf{0 . 5}$ & $\mathbf{0 . 6}$ & $\mathbf{0 . 7}$ & $\mathbf{0 . 8}$ & $\mathbf{0 . 9}$ \\
\hline $\boldsymbol{f}(\boldsymbol{x})$ & 0.009877 & 0.009874 & 0.009873 & 0.009873 & 0.009873 & 0.009873 & 0.009873 & 0.00987 & 0.00987 \\
Best & 0.009877 & 0.009874 & 0.009873 & 0.009873 & 0.009873 & 0.009873 & 0.009873 & 0.00987 & 0.00987 \\
Mean & 0.009975 & 0.009939 & 0.009885 & 0.009881 & 0.009876 & 0.009873 & 0.009873 & 0.00987 & 0.00987 \\
Worst & 0.010185 & 0.010174 & 0.009927 & 0.009937 & 0.00989 & 0.009874 & 0.009873 & 0.00987 & 0.00987 \\
Std. Dev. & $8.99 \mathrm{E}-05$ & $8.53 \mathrm{E}-05$ & $1.52 \mathrm{E}-05$ & $1.88 \mathrm{E}-05$ & $5.83 \mathrm{E}-06$ & $4.39 \mathrm{E}-07$ & $1.44 \mathrm{E}-07$ & $1.12 \mathrm{E}-09$ & $1.30 \mathrm{E}-07$ \\
\hline
\end{tabular}

TABLE 4: SIMULATION RESULTS OF SPEED REDUCER DESIGN FOR MR = 0.1 TO 0.9

\begin{tabular}{|c|c|c|c|c|c|c|c|c|c|}
\hline MR & 0.1 & 0.2 & 0.3 & 0.4 & 0.5 & 0.6 & 0.7 & 0.8 & 0.9 \\
\hline$f(x)$ & 2997.058 & 2997.058 & 2997.058 & 2997.058 & 2997.058 & 2997.058 & 2997.058 & 2997.058 & 2997.058 \\
\hline Best & 2997.058 & 2997.058 & 2997.058 & 2997.058 & 2997.058 & 2997.058 & 2997.058 & 2997.058 & 2997.058 \\
\hline Mean & 2997.058 & 2997.058 & 2997.058 & 2997.058 & 2997.058 & 2997.058 & 2997.058 & 2997.058 & 2997.058 \\
\hline Worst & 2997.058 & 2997.058 & 2997.058 & 2997.058 & 2997.058 & 2997.058 & 2997.058 & 2997.058 & 2997.058 \\
\hline Std. Dev. & $1.56 \mathrm{E}-11$ & 8.09E-12 & $4.42 \mathrm{E}-12$ & $4.24 \mathrm{E}-12$ & $3.45 \mathrm{E}-12$ & $1.25 \mathrm{E}-12$ & $2.23 \mathrm{E}-12$ & $4.31 \mathrm{E}-13$ & $8.13 \mathrm{E}-13$ \\
\hline
\end{tabular}



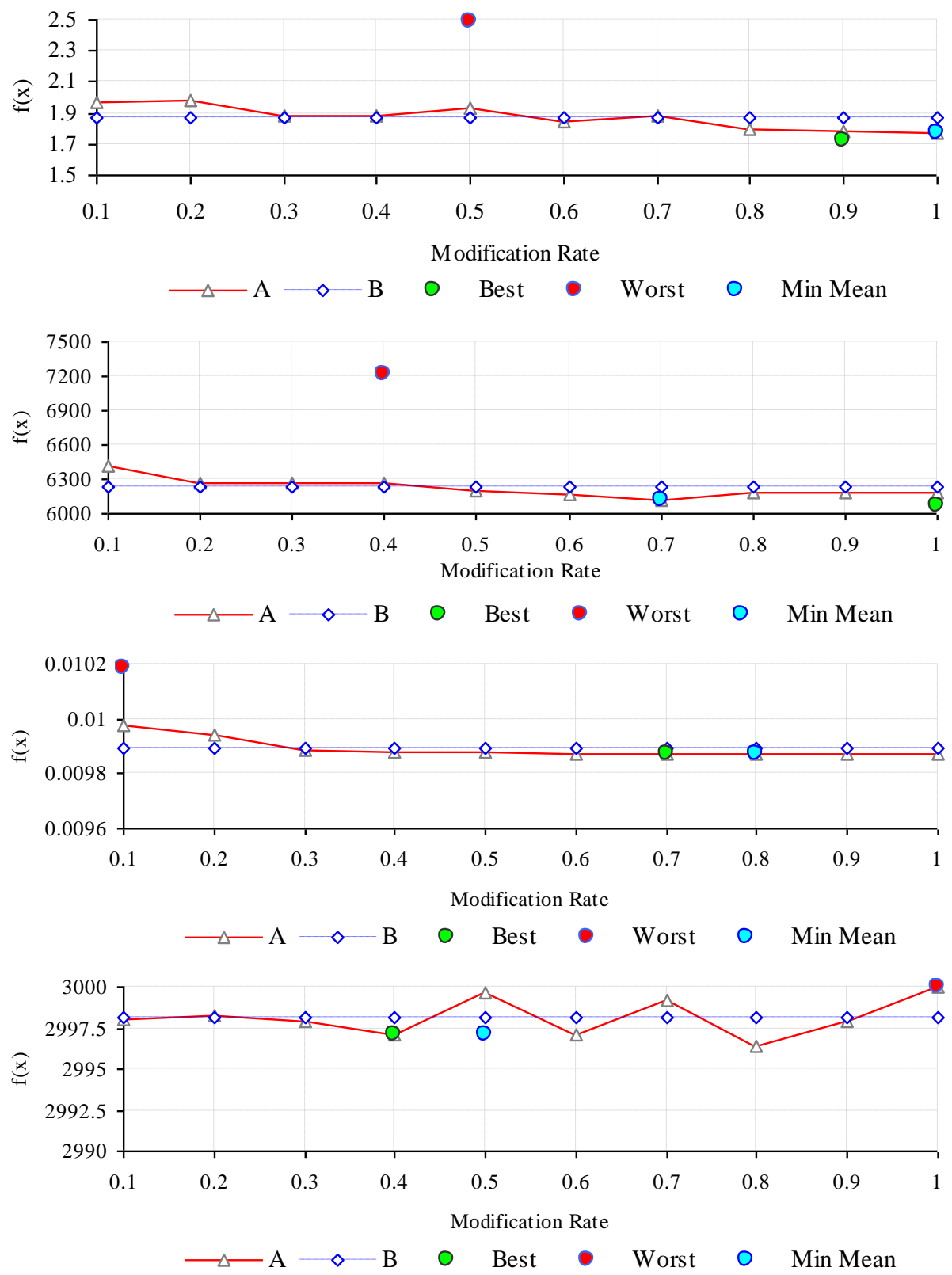

Figure 3. Fitness values of the problems are plotted against modification rate for the (a) Welded beam design (2) Pressure Vessel (c) Tension Compression Spring and (d) Speed Reducer design

\section{Conclusions}

According In constrained ABC user defined control parameter called modification rate (MR), is introduced that decides stochastically whether a memorized food source should be retained. In the present study we tried to analyze the impact of modification rate by gradually increasing it from 0.1 through 1.0. A preliminary conclusion is made that it is beneficial to set the modification rate to lower value so that the bee will search for a new food source in the closer neighborhood.

\section{References}

[1] D. Karaboga, An Idea Based On Honey Bee Swarm for Numerical Optimization, Technical Report TR06, Erciyes University, Engineering Faculty, Computer Engineering Department, 2005.

[2] Karaboga D, Basturk B, On the performance of artificial bee colony (ABC) algorithm, Applied Soft Computing, Volume 8, No. 1568-4946, 2007, pp. 687-697.

[3] Karaboga D, Basturk B, A powerful and efficient algorithm for numerical function optimization: artificial bee colony (ABC) algorithm. J. of Global Optimization, Volume 39, No. 0925-5001, 2007, pp. 459-471. 
[4] D. Karaboga, B. Akay, C. Ozturk, Modeling decisions for artificial intelligence, Artificial Bee Colony (ABC) Optimization Algorithm for Training FeedForward Neural Networks, LNCS 4617/2007, Springer-Verlag, 2007, pp. 318-329.

[5] C. Ozturk, D. Karaboga, Classification by neural networks and clustering with artificial bee colony (ABC) algorithm, in: Sixth International Symposium on Intelligent and Manufacturing Systems Features, Strategies and Innovation (Sakarya, Turkiye), October 14-17, 2008.

[6] B. Akay, D. Karaboga, A modified Artificial Bee Colony algorithm for real-parameter optimization, Inform Sci. (2010), doi:10.1016/j.ins.2010.07.015.

[7] N. Karaboga, A new design method based on artificial bee colony algorithm for digital iir filters, Journal of The Franklin Institute 346 (4) (2009) 328-348.

[8] Mustafa Sonmez, Artificial Bee Colony algorithm for optimization of truss structures, Applied Soft Computing, Volume 11, Issue 2, March 2011, Pages 2406-2418.

[9] Dervis Karaboga, Celal Ozturk, Nurhan Karaboga, Beyza Gorkemli, Artificial bee colony programming for symbolic regression, Information Sciences, Volume 209, 20 November 2012, Pages 1-15

[10] Nurhan Karaboga, Fatma Latifoglu, Adaptive filtering noisy transcranial Doppler signal by using artificial bee colony algorithm, Engineering Applications of Artificial Intelligence, Volume 26, Issue 2, February 2013, Pages 677-684.

[11] Francisco J. Rodriguez, M. Lozano, C. GarcíaMartínez, Jonathan D. González-Barrera, An artificial bee colony algorithm for the maximally diverse grouping problem, Information Sciences, Volume 230, 1 May 2013, Pages 183-196.

[12] Fei Kang, Junjie Li, Haojin Li, Artificial bee colony algorithm and pattern search hybridized for global optimization, Applied Soft Computing, Volume 13, Issue 4, April 2013, Pages 1781-1791.

[13] Dervis Karaboga, Beyza Gorkemli, Celal Ozturk, Nurhan Karaboga, A comprehensive survey: artificial bee colony (ABC) algorithm and applications, Artificial Intelligence Review, Doi: 10.1007/s10462-012-9328-0.

[14] D. Karaboga, B. Basturk, Advances in soft computing: foundations of fuzzy logic and soft computing, in: Artificial Bee Colony (ABC) Optimization Algorithm for Solving Constrained Optimization Problems, LNCS, 4529/2007, Springer-Verlag, 2007, pp. 789-798.

[15] Deb K, An Efficient Constraint-handling Method for Genetic Algorithms, Computer Methods in Applied Mechanics and Engineering, Volume 186, No. 0045-7825, 2000, pp. 311-338.

[16] G. V. Reklaitis, A. Ravindran, and K. M. Ragsdell. Engineering Optimization Methods and Applications. New York: Wiley, 1983.

[17] Coello Coello CA. Use of a self-adaptive penalty approach for engineering optimization problems. Comput Ind, 2000; 41(2):113-27.

[18] J. Golinski. An adaptive optimization system applied to machine synthesis. Mechanism and Machine Synthesis. 8(1973), pages 419-436, 1973.

[19] Belegundu AD. A Study of Mathematical Programming Methods for Structural Optimization, PhD thesis, Department of Civil and Environmental Engineering, University of Iowa, Iowa, USA, 1982.

\section{Appendix - I}

\section{Welded Beam Design}

Minimizes the cost of the beam subject to constraints on shear stress, $\tau$, bending stress in the beam, $\sigma$, buckling load on the bar, $P_{c}$, end deflection of the beam, $\delta$, and side constraints. This problem consists of a nonlinear objective function, five nonlinear and two linear inequality constraints. The solution is located on the boundaries of the feasible region. The ratio of feasible region to entire search space is quite small for welded beam problem.

There are four design parameters $x_{1}, x_{2}, x_{3}$ and $x_{4}$ correspond to $h, l, t$ and $b$ variables, respectively, shown in Fig.A1.

$$
\begin{aligned}
& \min _{x} f(X)=1.10471 x_{1}^{2} x_{2}+0.04811 x_{3} x_{4}\left(14.0+x_{2}\right) \\
& \text { Subject to } g_{1}(X): \tau(x)-\tau_{\max } \leq 0 \\
& g_{2}(X): \sigma(x)-\sigma_{\max } \leq 0 \\
& g_{3}(X): x_{1}-x_{4} \leq 0 \\
& g_{4}(X): 1.10471 x_{1}^{2}+0.04811 x_{3} x_{4}\left(14.0+x_{2}\right)-5.0 \leq 0 \\
& g_{5}(X): 0.125-x_{1} \leq 0 \\
& g_{6}(X): \delta(x)-\delta_{\max } \leq 0 \\
& g_{7}(X): P-P_{c}(x) \leq 0
\end{aligned}
$$




$$
\begin{aligned}
& \text { where } \quad \tau(X)=\sqrt{\left(\tau^{\prime}\right)^{2}+2 \tau^{\prime} \tau^{\prime \prime} \frac{x_{2}}{2 R}+\left(\tau^{\prime \prime}\right)^{2}} \text {, } \\
& \tau^{\prime}=\frac{P}{\sqrt{z} x_{1} x_{2}} \\
& \tau^{\prime \prime}=\frac{M R}{J} \\
& M=P\left(L+\frac{x_{2}}{2}\right) \\
& R=\sqrt{\frac{x_{2}^{2}}{4}+\left(\frac{x_{1}+x_{3}}{2}\right)^{2}} \\
& J=2\left\{\frac{x_{1} x_{2}}{\sqrt{2}}\left[\frac{x_{2}^{2}}{12}+\left(\frac{x_{1}+x_{3}}{2}\right)^{2}\right]\right\} \\
& \sigma(X)=\frac{6 P L}{x_{4} x_{3}^{2}} \\
& \delta(X)=\frac{4 P L^{3}}{E x_{3}^{3} x_{4}} \\
& P_{c}=\frac{4.013 E \sqrt{x_{3}^{2} x_{4}^{6}}}{L^{2}}\left(1-\frac{x_{3}}{2 L} \sqrt{\frac{E}{4 G}}\right)
\end{aligned}
$$

and $\mathrm{P}=6000 \mathrm{lb}$., $\mathrm{L}=14$ in., $\delta_{\max }=0.25$ in., $\mathrm{E}=30 \times 10^{6} \mathrm{psi}, \mathrm{G}=12 \times 10^{6} \mathrm{psi}, \tau_{\max }=1,3600 \mathrm{psi}, \sigma_{\max }=3,0000 \mathrm{psi}$, $\mathrm{X}=\left(x_{1}, x_{2}, x_{3}, x_{4}\right)^{\mathrm{T}}, 0.1 \leq x_{1}, x_{4} \leq 2.0,0.1 \leq x_{2}, x_{3} \leq 10$.

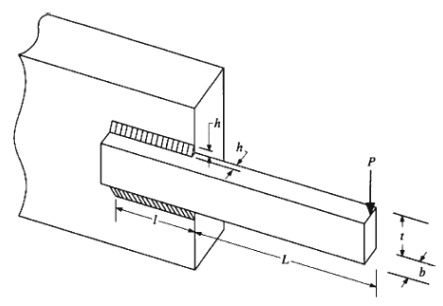

Figure A1. The welded beam problem

\section{Pressure Vessel Problem}

The pressure vessel problem is to minimize the total cost of material, forming and welding of a cylindrical vessel shown in Fig.A2. Pressure vessel problem has a nonlinear objective function, a nonlinear and three linear inequality constraints. Since the problem has two discrete variables and two continuous variables, it is a mixed discrete-continuous constrained optimization problem. The four design variables are $x_{1}$ (thickness TS of the shell), $x_{2}$ (thickness TH of the head), $x_{3}$ (inner radius $\mathrm{R}$ ) and $x_{4}$ (length $\mathrm{L}$ of the cylindrical section of the vessel, not including the head). $x_{1}$ and $x_{2}$ are to be in integral multiples of 0.0625 inch which are the available thicknesses of rolled steel plates. The radius $x_{3}$ and the length $x_{4}$ are continuous variables.

$$
\begin{aligned}
& \min _{X} f(X)=0.6224 x_{1} x_{2} x_{3}+1.7781 x_{2} x_{3}^{2}+3.1661 x_{1}^{2} x_{4}+19.84 x_{1}^{2} x_{3} \\
& \text { Subject to } \quad g_{1}(X):-x_{1}+0.0193 x_{3} \leq 0 \\
& g_{2}(X):-x_{2}+0.00954 \leq 0 \\
& g_{3}(X):-\pi x_{3}^{2} x_{4}-\frac{4}{3} \pi x_{3}^{3}+1296000 \leq 0 \\
& g_{4}(X): x_{4}-240 \leq 0
\end{aligned}
$$

where $X=\left(x_{1}, x_{2}, x_{3}, x_{4}\right)^{\mathrm{T}}$. The ranges of the design parameters are $0 \leq x_{1}, x_{2} \leq 99,10 \leq x_{3}, x_{4} \leq 200$.

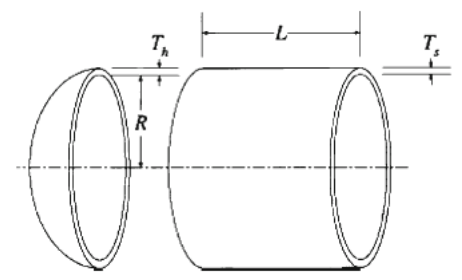

Figure A2. The pressure vessel problem 


\section{Speed Reducer Design}

The aim of the speed reducer design is to minimize the weights of the speed reducer, Fig. A3, subject to constraints on bending stress of the gear teeth, surface stress, transverse deflections of the shafts and stresses in the shafts. Speed reducer problem has seven nonlinear and four linear constraints. Four constraints are active at the best known feasible solution [3.5000 0.700017 .00007 .30007 .71533 .35025 .2867 ] producing a $2994.34 \mathrm{~kg}$ gearbox.Design parameters of the speed reducer problem, the face width $(b)$, module of teeth $(m)$, number of teeth in the pinion $(z)$, length of the first shaft between bearings $\left(l_{1}\right)$, length of the second shaft between bearings $\left(l_{2}\right)$ and the diameter of the first shaft $\left(d_{1}\right)$ and second shaft $\left(d_{2}\right)$ correspond to $x_{1}, x_{2}, \ldots, x_{7}$, respectively. Some optimization algorithms have been reported to have difficulties in finding the feasible space and is an example of a mixed integer programming problem. The third variable (number of teeth) is of integer value while all other variables are continuous.

$$
\begin{aligned}
& \min _{X} f(X)=0.7854 x_{1} x_{2}^{2}\left(3.3333 x_{3}^{2}+14.9334 x_{3}-43.0934\right)-1.508 x_{1}\left(x_{6}^{2}+x_{7}^{2}\right)+7.4777\left(x_{6}^{3}+x_{7}^{3}\right) \\
& \text { Subject to } \quad g_{1}(X): \frac{27}{x_{1} x_{2}^{2} x_{3}}-1 \leq 0 \\
& g_{2}(X): \frac{397.5}{x_{1} x_{2}^{2} x_{3}}-1 \leq 0 \\
& g_{3}(X): \frac{1.93 x_{4}^{3}}{x_{2} x_{3} x_{6}^{4}}-1 \leq 0 \\
& g_{4}(X): \frac{1.93 x_{5}^{3}}{x_{2} x_{3} x_{7}^{4}}-1 \leq 0 \\
& g_{5}(X): \frac{\left(\left(\frac{745 x_{4}}{x_{2} x_{3}}\right)^{2}+157.5 \times 10^{6}\right)^{1 / 2}}{110.0 x_{6}^{3}}-1 \leq 0 \\
& g_{6}(X): \frac{\left(\left(\frac{745 x_{4}}{x_{2} x_{3}}\right)^{2}+157.5 \times 10^{6}\right)^{1 / 2}}{85.0 x_{7}^{3}}-1 \leq 0 \\
& g_{7}(X): \frac{x_{2} x_{3}}{40}-1 \leq 0 \\
& g_{8}(X): \frac{5 x_{2}}{x_{1}}-1 \leq 0 \\
& g_{9}(X): \frac{x_{1}}{12 x_{2}}-1 \leq 0 \\
& g_{10}(X): \frac{1.5 x_{6}+1.9}{x_{4}}-1 \leq 0 \\
& g_{11}(X): \frac{1.1 x_{7}+1.9}{x_{5}}-1 \leq 0
\end{aligned}
$$

where $2.6 \leq x_{1} \leq 3.6,0.7 \leq x_{2} \leq 0.8,17 \leq x_{3} \leq 28,7.3 \leq x_{4} \leq 8.3,7.8 \leq x_{5} \leq 8.3,2.9 \leq x_{6} \leq 3.9,5.0 \leq x_{7} \leq 5.5$.

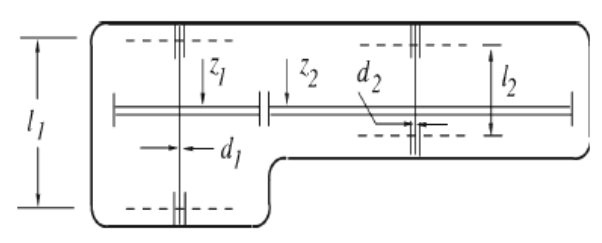

Figure A3. The speed reducer problem

\section{Tension/Compression Problem}

This problem deals with minimizing of the weight of the tension/compression spring shown in Fig. A4, subject to constraints on the minimum deflection, shear stress, surge frequency, diameter and design variables. This problem has a nonlinear objective function, a linear and three nonlinear inequality constraints.

The design variables are the wire diameter, $\mathrm{d}(\mathrm{x} 1)$, the mean coil diameter, $\mathrm{D}(\mathrm{x} 2)$, and the number of active coils, $\mathrm{N}(\mathrm{x} 3)$. The problem is formulated as: 


$$
\begin{aligned}
& \min _{X} f(X)=(N+2) D d^{2} \\
& \text { Subject to } \\
& g_{1}(X): 1-\frac{D^{3} N}{71785 d^{4}} \leq 0 \\
& g_{2}(X): \frac{4 D^{2}-d D}{12566\left(D d^{3}-d^{4}\right)}+\frac{1}{5108 d^{2}}-1 \leq 0 \\
& g_{3}(X): 1-\frac{140.45 d}{D^{2} N} \leq 0 \\
& g_{4}(X): \frac{D+d}{1.5}-1 \leq 0
\end{aligned}
$$

$X=(d, D, N)^{\mathrm{T}}, 0.05 \leq d \leq 2.0,0.25 \leq D \leq 1.3,2.0 \leq N \leq 15.0$

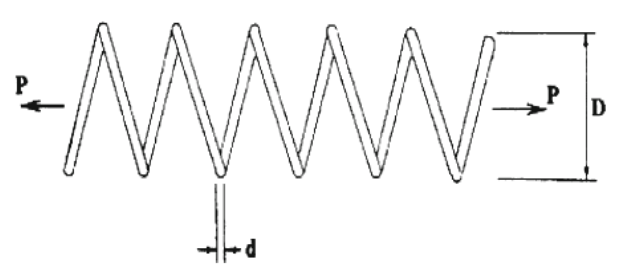

Figure A4. The tension/compression spring problem

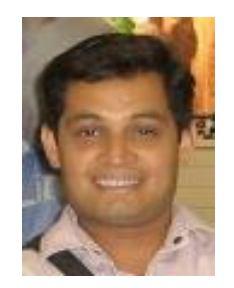

Tarun Kumar Sharma: Assistant Professor in Amity Institute of Information Technology, Amity University Rajasthan, India. His research areas are evolutionary and Swarm intelligence algorithms and their applications in Software Engineering. He is in Editorial Board and reviewer of many refereed Journals. He has published about 40 research papers in Journal of repute and in refereed international Journals.

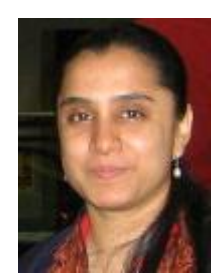

Millie Pant: Millie Pant: Associate Professor, Indian Institute of Technology, Roorkee, India. She has published above 200 research publications in referred journals and international conferences. She has been keynote speakers to various seminars, conferences and development programs. Her key research areas are Evolutionary Computing, Swarm Intelligence and their application in various areas of Engineering.

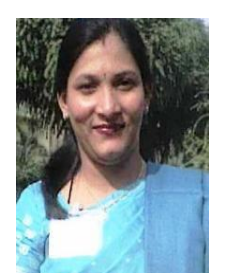

Deepshikha Bhargava: She has more than 15 years of teaching experience. At present she is heading Amity Institute of Information Technology, Amity University Rajasthan, and Jaipur. She has overall 16 book publications into her credit and one under publication. She has presented and published more than 30 research papers in International \& National Journals and Conferences. She is Member of International Association of Computer Science and
Information Technology (IACSIT) Singapore, International Computer Science Teachers Association (CSTA), ACM-USA, Computer Society of India (CSI), Indian Society of Lightening Engineers (ISLE), India. She has also member of Reviewer \& Editorial Board of International and National Journals: "International Journal of Science and Research (IJSR)", "International Journal of Advanced and Innovative Research (IJAIR)", "International Journal of Computing (JCT)", "International Journal of Engineering Associates (IJEA)", "International Journal of Emerging Technologies and Applications in Engineering, Technology and Sciences (IJETAETS)", "International Journal of Computer Applications in Engineering, Technology and Sciences (IJCAETS)", "Blue Ocean Research Journal (BORJ)" and "International Journal of Soft Computing and Engineering (IJSCE)”.. 